\title{
Impact of Pharmacy Services on Time to Elexacaftor-Tezacaftor-Ivacaftor Initiation
}

\author{
Lauren Roder ${ }^{1}$, Michelle Simonsen $^{1}$, Lindsey Fitzpatrick ${ }^{1}$, Jennifer Loucks ${ }^{1}$, and Jianghua \\ $\mathrm{He}^{1}$ \\ ${ }^{1}$ The University of Kansas Health System
}

December 12, 2021

\begin{abstract}
The approval of elexacaftor-tezacaftor-ivacaftor (ELX/TEZ/IVA) expanded highly effective cystic fibrosis transmembrane receptor modulator therapy to approximately $90 \%$ of persons age 12 and older with cystic fibrosis. Clinical pharmacists and pharmacy technicians played a key role in planning for ELX/TEZ/IVA initiation prior to FDA approval as well as initiating therapy after approval. This study evaluates the impact of pharmacy services on time to ELX/TEZ/IVA initiation. A retrospective chart review evaluated patients qualifying for ELX/TEZ/IVA at a single health system between October 21, 2019 and April 1, 2020. Patients filling ELX/TEZ/IVA at an integrated health system specialty pharmacy (HSSP) versus an outside specialty pharmacy (SP) started on therapy an average of 10.8 days faster (10.8 days \pm 14.0 vs 21.6 days \pm 18.8 respectively; $\mathrm{p}=0.006)$. More patients filling at a HSSP received ELX/TEZ/IVA within 14 days of the prescription being written compared to outside SPs (82.0\% vs $41.4 \%$ respectively; $\mathrm{p}=0.001)$. Pre-ELX/TEZ/IVA initiation, patients were hospitalized for a CF related complication for an average of 6.26 days (range 0-183) compared to 1.16 days (range 0-91) post-ELX/TEZ/IVA initiation. Lastly, an estimated $\$ 134,810$ was saved in the 105 patients that were able to fill ELX/TEZ/IVA at a HSSP by initiating drug an average of 10.8 days quicker than outside SPs. The results of this study demonstrate the value of an integrated HSSP model. Further advocacy for inclusion of integrated HSSPs by pharmacy benefit managers is needed to optimize medication access, control costs, and improve patient outcomes for patients receiving care within a health system.
\end{abstract}

\section{Hosted file}

ELX-TEZ-IVA Manuscript_L Roder_Final_no tables \& figures.docx available at https://authorea. com/users/450776/articles/549059-impact-of-pharmacy-services-on-time-to-elexacaftortezacaftor-ivacaftor-initiation 

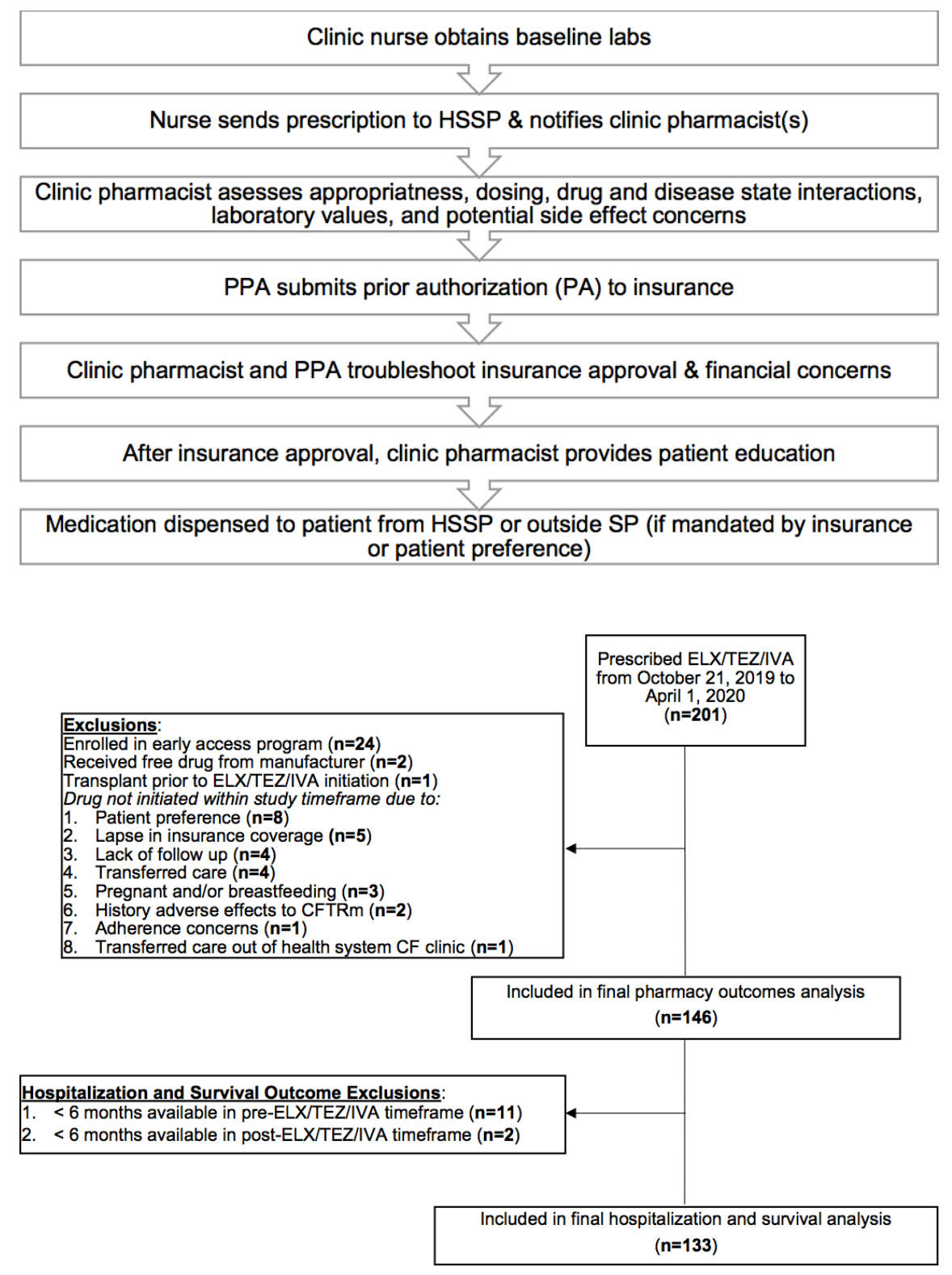

\section{Hosted file}

Table I.docx available at https://authorea.com/users/450776/articles/549059-impact-ofpharmacy-services-on-time-to-elexacaftor-tezacaftor-ivacaftor-initiation 

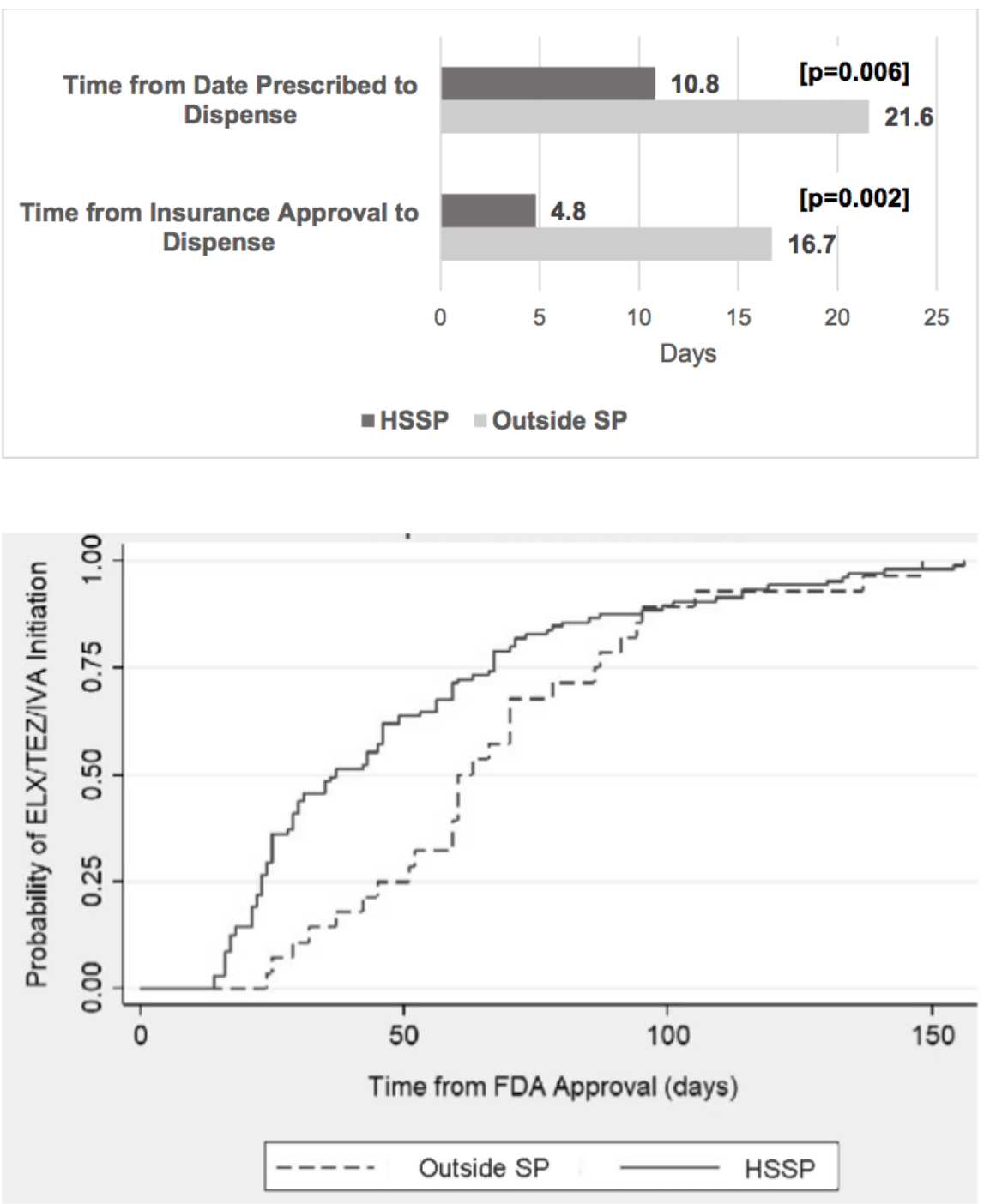